\title{
MUSICtable: A Map-based Ubiquitous system for Social Interaction with a digital music Collection
}

\author{
Jennifer Gluck \\ Dept. of Computer Science \\ University of British Columbia \\ 2366 Main Mall \\ Vancouver, BC V6T 1 Z4 \\ gluck@cs.ubc.ca
}

\author{
Ian Stavness \\ HCT Laboratory \\ University of British Columbia \\ 2356 Main Mall \\ Vancouver, BC, V6T 1 Z4 \\ stavness@ece.ubc.ca
}

\author{
Leah Vilhan \\ HCT Laboratory \\ University of British Columbia \\ 2356 Main Mall \\ Vancouver, BC, V6T 1 Z4 \\ leahv@ece.ubc.ca
}

\begin{abstract}
Popular acceptance of the mp3 digital music standard has greatly increased the complexity of organizing and playing large music collections. Existing digital music systems do not adequately support exploration of a collection, nor do they cater to multi-user interaction in a social setting. In this paper, we present the design of a ubiquitous system that utilizes spatial visualization to support exploration and social interaction with a large music collection. We implemented a prototype as a proof of concept of our design. Initial user testing demonstrates that our system encourages participation and strengthens social cohesion. Our work contributes to interactive interface research in that it extends the utility of map-based visualization of digital music.
\end{abstract}

\section{Keywords}

Social interaction, ubiquitous computing, mp3, digital media, music map, tabletop display.

\section{INTRODUCTION}

Music recording is arguably one of the most culturally important modern human inventions. Advancement in digital recording technology and the mp3 digital music standard have spawned a trend of large music collections. With this new technology has come a novel set of problems. An important issue is that interfaces that are used to play digital music do not support large collections in a social situation.

Traditional forms of recorded music such as compact discs (CDs), tapes, and records present music at the granularity of the album a set of 10-15 songs. Digital media poses more challenging organization problems because $\mathrm{mp} 3$ files reduce the granularity of music to the size of the individual song. It is much more difficult to deal with a collection of $600 \mathrm{mp} 3$ files than the collection of 50 CDs from which the songs were ripped.

Digital solutions to the music organization problem have been attempted by means of metadata (ID3 tags) that explicitly denote

Permission to make digital or hard copies of all or part of this work for personal or classroom use is granted without fee provided that copies are not made or distributed for profit or commercial advantage and that copies bear this notice and the full citation on the first page. To copy otherwise, or republish, to post on servers or to redistribute to lists, requires prior specific permission and/or a fee.

HIT’04, Dec. 13, 2004, Vancouver, British Columbia, Canada. the artist, album name, year of production, and genre of an $\mathrm{mp} 3$ file. In practice, these fields are rarely filled in correctly and there is no universal standard in genre classification. Thus, classification and of large digital collections remains an open problem for both single users interacting with a music player, as well as in the domain of multi-user social interaction with recorded music.

The standard PC-based digital music player is specifically designed for single-user interaction. The user interacts with the system by adding individual mp3 files to a playlist. Selection of each file requires linear searching within the computer file system. The playlist metaphor does not support the idea of a continuous mood or feeling of music - often the goal of the music listener because selection occurs at the granularity of a particular song. Additionally, this selection granularity may be problematic for users who are not familiar with the specific artist and song names. Furthermore, the system is constrained to the computer itself. These characteristics result in a system that requires a nontrivial investment of interaction time by a single user. A trend that we found through an initial user study was that many users abandoned all direct control for the music player by engaging a "random" play mode with all songs in the collection. This method requires continuous interaction with the system as users generally will continue to skip to a new random song until they find one that fits their current mood.

The single user interface of the PC-based digital music player clearly does not support music selection by multiple people in a social situation. One manifestation of this deficiency is a phenomenon we will refer to as "separate party syndrome:" A small number of people tend to gather around the desktop computer, away from the other attendees of the party, dominating the selection of music. Only a small fraction of the people participates in music selection with this type of interface and those who do are removed from the social atmosphere of the party while engaged in music selection. Another compounding factor reducing participation of music selection in a social setting is granularity. Users are often unfamiliar with another person's music collection and therefore are less likely to make song-by-song selections at a party.

Effective social interaction with a digital collection requires a means to explore and discover its contents, and to play music in a manner that is inclusive of the preferences of each individual yet results in a smooth progression of music through different moods, styles, and tempos of music. Such a solution should not require any substantial investment of interaction time, nor should it require a departure from the social atmosphere. 
In an effort to address the shortcomings of current digital music solutions, we present a ubiquitous system that utilizes spatial visualization in order support exploration and social interaction with a large music collection.

\section{RELATED WORK}

In this section we discuss research directions that have influenced our design. This work falls into four categories: visualization of digital music collections, social interaction and music, interactive displays, and ludic design.

\subsection{Spatial Visualization of Music}

As personal digital music collections grow larger and larger, the science of visualizing such archives is becoming popular. A number of papers on this topic have been published. For example, Torrens et al. [7] have experimented with visualization techniques such as disk, rectangle, and tree-map visualization.

Other research has taken on the analogy of cartography. Pampalk et al. [5] have created a map-based visualization called Islands of Music. This system creates a self organizing map by sampling the songs and extracting raw audio data. The music is classified by comparing traits such as loudness, sensation, and rhythm patterns. The results are used to display the music collection spatially, such that similar pieces of music appear close to each other. This organization is visualized as a geographic map.

Similarly, van Gulik et al. [2] have developed the "Artist Map," a technique that employs graph-drawing algorithms based on an energy model. Like Islands of Music, this system presents a visualization using data derived from the music itself, but also incorporates metadata that can be obtained from ID3 tags and web services.

In all the visualization systems researched, the objective is to display a representation of the music collection. These systems focus on navigation and exploration of a music collection. We have yet to find evidence of any of these systems being utilized to listen to the music library, for example, via the creation of playlists. We endeavor to extend the idea of map-based music visualization systems by using such a visualization to support the selection and playing of music.

\subsection{Social Interaction and Music}

Current research on social interaction and music has focused on the creation of music through social interaction. A project at the MIT Media Lab examined changing ambient music at a social gathering based on the type of drinks people were drinking [3]. Ambient music was not recorded music, but a combination of various musical sounds.

In the domain of recorded music, O'Hara et al. have examined a song selection system used in a public space [4]. Patrons of a pub democratically chose which song to play next. This is a different social context, as our interface is intended for more private social gatherings. Furthermore, our interface will gather input from each every user's selection, incorporating this data to play music that will cater to the preferences of all participating users. This is quite different from a voting mechanism for song selection on a songby-song basis.

\subsection{Ubiquity and Interactive Displays}

Rogers and Lindley [6] conducted a study to investigate how the physical orientation of a shared display affects group collaboration. They found that table displays encourage group members to switch roles often, explore ideas, and closely follow along with what each member of the group was doing. In contrast, groups find collaboration around a wall display to be socially awkward, and usually designate one leader to interact with the display.

The social aspects surrounding the use of tables make them appealing for use as displays. Tables are common and incorporated into the design of most rooms, allowing tabletop displays to blend into everyday life. This "blending" is a defining characteristic of ubiquitous computing, presented by Weiser [8]. Based on these findings, we feel that a tabletop display is an ideal medium to utilize in separating the music collection from the computer. We were also encouraged by the social affordances associated with tables, and believe that they will lend well to creating a system where groups of people are encouraged to interact together.

\subsection{Ludic Design}

Gaver et al. explored the notion of ludic design in order to address computer use in the realm of people as "playful creatures" ("Homo Ludens") [1]. The Drift Table is a tabletop display that displays aerial photography. The display slowly drifts across a landscape and is controlled by the distribution of weight on its surfaces. Goals derived from the ludic outlook include avoiding the appearance of a computer and supporting social engagement in ludic activities. Strongly contrasting with traditional goals for computer systems, the designers' objective was to avoid meeting users' immediate desires or demands. For instance, the system was designed to disallow a move to a particular location. Instead, users work together, placing different objects on the tabletop, to influence the direction of the drift. We were inspired by this idea of influence over selection in order to support open-ended, social use of an interface.

\section{DESIGN}

In the quest for a system that supports exploration and social interaction with a large digital music collection, our general design goals were as follows:

- Separate the music collection from the computer.

- Preserve social cohesion.

- Present the collection in a form that supports exploration.

- Encourage collaboration in the music selection process.

We propose a table-based system that utilizes music map visualization. An area of selection is displayed on the map, and corresponds to the location of the song that is currently playing. Users interact with the system by entering directional input to affect the location of the selection area.

The notion of exploration in a musical space is important our design. Users who are unfamiliar with a particular music collection may be timid and thus shy away from participating in music selection. By providing an interface that suggests traversal and exploration, everyone is at an equal level of understanding. This allows the unfamiliar user the freedom to participate and influence the navigation of the map without social pressures. We 
aim to provide an interface in which there is no one person choosing a specific song. Instead, a group of people work together to influence the musical atmosphere of a room by exploring a map.

A tabletop system, when strategically placed in a room, ensures that social cohesion is preserved. First, this type of system ensures that users are not required to remove themselves physically from a social area in order to attend to a computer, which may be located in the corner of the room or in another room entirely. Second, by separating our system from a traditional PC, we ensure that users will not become distracted by other software while in the process of selecting music. As discussed above, tabletop systems are also known to encourage collaboration.

Because of the low level granularity of digital music collections, displaying the collection at the level of a song can make the selection process tedious. Displaying a collection as a series of folders organized by traits such as artist or genre is not ideal. Instead, we aim to separate the names and titles of songs from the visualization, instead focusing on the mood or atmosphere inspired. We felt that the geographic map metaphor addressed this goal. This idea of a "music map" visualization of a music collection is useful and engaging because it compels users to traverse and explore the music. Just as an orienteer navigates a map to discover new locations, our users will be able to discover new music. We decided to implement our system as a static map with a moving selection point as opposed to the Drift Table design, which utilizes a static selection point and a moving map. This provides users with the context necessary to not just explore, but to learn and retain the discovered information.

Simultaneous multiple-user input is also important in encouraging collaboration. However, such input cannot be discrete: input from each user must be multiplexed together to achieve the music selection. We capitalized on the idea of influence over selection broached by Gaver et al. [1]. The different directional input entered by each user are combined together to influence the direction of movement of the selection area. The idea of influence is also important in effectively matching the interaction style to the map metaphor. In the case of a music system, 'selection' gives the user the expectation of specifying the exact song to be played. This does not work well in a group setting: each person will want to hear 'his' or 'her' specific song, and unfamiliar users go without a say. With an 'influence' semantic, on the other hand, there is no such expectation or limitation. Instead, users are given the sense that their actions will have an impact on the music played, but without the certainty of an exact song. Also of note is the fact that the map visualization, devoid of artist and song names, does not give the option of selecting a specific song; thus, we must approach the user interaction from the influence perspective.

\section{PROTOTYPE}

The short time frame of the project forced our group to refine our prototype design and reduce the scope to a few of the issues we found surrounding the design of a social music player interface. We believe that the larger idea of spatial visualization as a novel interface has a broader scope than the specific manifestation that we developed in our prototype. Please refer to the Future Work section for a survey of our other design ideas.

\subsection{Prototype Design}

\subsubsection{The Music Map}

We created our own music map for the prototype; however, our system design can support any music map visualization, such as those referenced in the related work section. In the creation of our map, 393 songs of various styles were subjectively sorted by similarity of sound and genre into 50 categories. The categories were then arranged spatially such that similar genres appear near one another. Each category is represented by a circle, the size of each circle proportional to the number of songs contained in the set. Different outlines were applied to the circles in an attempt to provide visual cues about the type of music contained. For example, softer, lighter sounds have a smoother edge: The classical "islands" were untouched, remaining true circles. The jazz islands have a slightly wavy texture. Harder rock categories were given a jagged appearance. Refer to Figure 1 for a screen capture of the music map visualization.

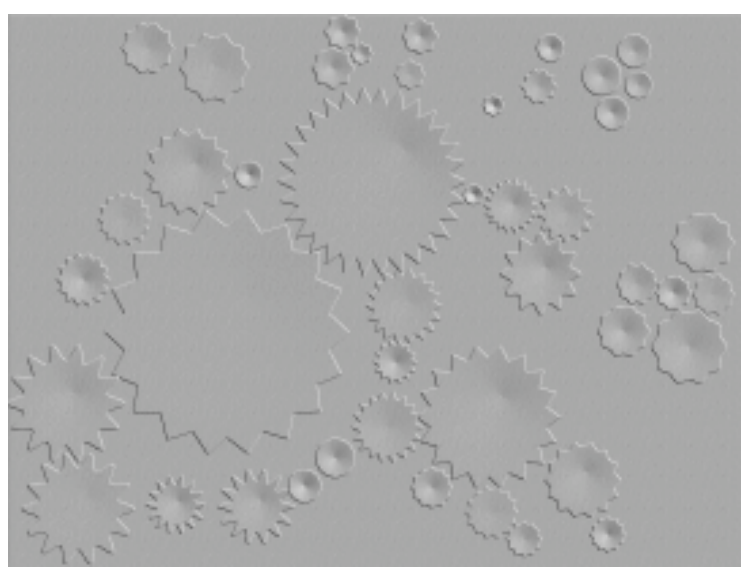

Figure 1: The MUSICtable music map.

\subsubsection{User Interface}

The map is presented on the tabletop display as a grayscale image. The area of selection is denoted by a small circular viewpoint that appears in colour. The notion of influence is attained through the analogy of a magnet. Eight buttons arranged at the compass points around the tabletop display allow users to "nudge" the position of the magnet widget in the direction of the button. LED indicators around the buttons, in addition to the movement of the magnet cursor, provide users with feedback that their button input has affected the system. The selection point is slowly pulled towards the magnet. This incremental position change fits well with the semantic of influence and it ensures that multiple people will be able to influence the direction and speed of music change. The music plays continuously. Each time a song ends, the next song to be played is selected from the island nearest to the current location of the selection point.

\subsection{Implementation}

\subsubsection{Software System}

The control software of the system was developed in $\mathrm{C}++$ with the openGL graphics library. We needed to implement two main tasks:

1. Select a song based on the location of the selection area 


\section{Ensure continuous music play}

In order to select a song based on location, all songs were loaded into a two-dimensional spatial subdivision data structure. The type of data structure chosen was a quad-tree, which creates a grid from the music map where each grid square contains at most five songs. When the system must select the next song to play, it derives which grid square the selection point is in and then randomly chooses a song from that grid square.

With this fine-grained data structure (five songs per node), nodes may exist without associated songs, resulting in gaps in music play. We solved to this problem by using multiple data structures with varying levels of detail. When choosing the song to play, the logic unit will first search the most finely grained data structure. If a song is not found, a more coarsely grained data structure is searched.

\subsubsection{Hardware System}

The main aspects of hardware implementation were the design of a tabletop display and the button hardware portion of the user interface. We used an LCD monitor embedded into the middle of a table in order to provide a bright display with high resolution. A glass cover over top of the display was flush with the table surface to maintain the affordances of a table - i.e. people could still set down drinks on the surface. The table stands three feet tall, a comfortable height to use while standing.

The button hardware was created with simple contact push buttons and LEDs. We used a Phidget Interface Kit 8/8/8 board to capture button input and to control the LED indicators. This interface board was housed inside of the table. The software system of the MUSICtable ran on a laptop computer that was also placed inside the MUSICtable. High quality computer speakers were used to play the music from the laptop computer.

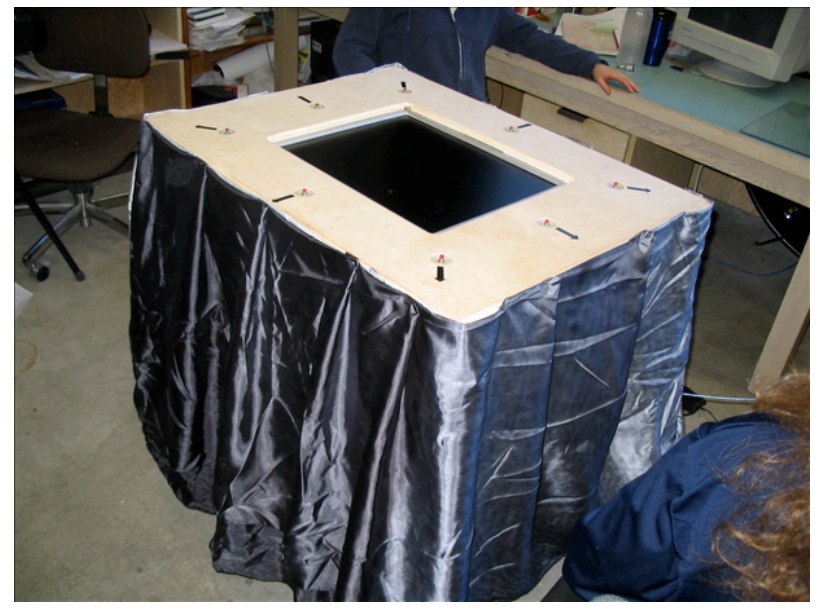

Figure 2: The MUSICtable prototype.

The table and button assemblies were all designed to maintain a high level of aesthetic appeal. The computer and hardware housed inside the table were kept out of view using a colorful drape. The prototype is shown in Figure 2.

\section{USER EVALUATION}

We evaluated our MUSICtable system in comparison with a laptop computer system running Nullsoft's mp3 software player
Winamp [9]. This software is commonly used to build and play playlists of songs from a digital music collection.

As mentioned above, a general goal of our system was to avoid the "separate party syndrome" often involved in digital music selection in a social setting. We felt it necessary to test our system against a Winamp system that was also located in the centre of the party room. This was in order to show that more is required to solve the problems associated with social interaction with a digital music collection than merely moving the traditional computer-based system into the centre of the social circle.

\subsection{Hypotheses}

H1: A larger percentage of participants will involve themselves in music selection with the MUSICtable tabletop system than with the PC-based Winamp system.

H2: The MUSICtable system will preserve social cohesion: Participants will experience lowered feelings of separation from the social atmosphere while involved in music selection with MUSICtable than with Winamp. The MUSICtable system will encourage a higher level of collaboration than Winamp.

\subsection{Participants}

Thirteen participants, all between the ages of 18 and 30, were recruited from a pool of graduate students. This age group is representative of the generation that has embraced digital music collections and related technologies.

\subsection{Procedure}

We utilized a within-subjects experimental design. There can be no system learning effects between the two levels, so counterbalancing is unnecessary.

The experiment consisted of a party to which the participants were invited. Snacks and refreshments were served to evoke a social "party" atmosphere. The experiment was divided into two 45minute sessions. Subjects were told to feel free to change the music that was playing; that is, music selection was in the hands of the party attendees.

During the first session, a laptop computer running Winamp was used as the music selection system. During the second session, our MUSICtable system was used. During session one, the laptop was placed on top of the table that housed the MUSICtable system, with the prototype turned off. This was done so that both systems were centrally located in the room. The switch between the two systems was made as inconspicuously as possible while the users' attention was directed to the other side of the room.

Results were collected via a post-party questionnaire. Here, participants indicated which of the two systems they interacted with (Winamp, MUSICtable, or both). Participants answered 


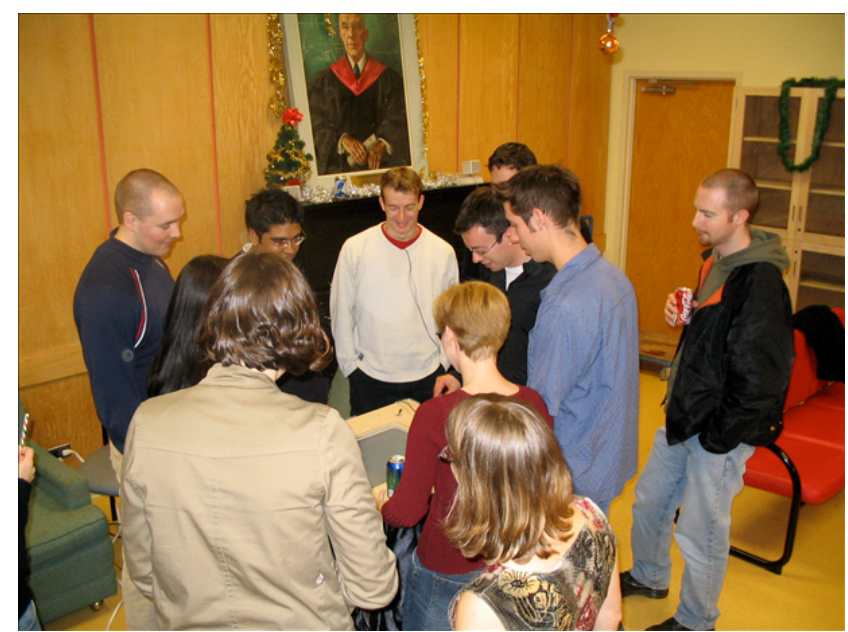

Figure 3: Participants interacting with the MUSICtable

questions pertaining to level of collaboration encouraged and feelings of separation induced by use of each system. Additionally, the survey gauged understanding of the system itself, as well as a preference between music player systems.

\section{RESULTS AND DISCUSSION}

\subsection{Verification of Hypotheses}

We observed that eight users participated in selection of music using Winamp, whereas twelve users participated in selection of music using MUSICtable. Only one user who used Winamp did not use MUSICtable. This supports our hypothesis that a larger number of participants will involve themselves in music selection with the MUSICtable tabletop system.

Qualitative results from the questionnaire indicated that users felt more encouraged to collaborate in music selection while using the MUSICtable than while using Winamp, and felt less separated from the social circle while involved in MUSICtable interaction:

The mean response to the survey question "The Winamp system encouraged me to collaborate with others while selection music" was 2.5 (corresponding to an answer of "Disagree-Neutral"). The mean response to the survey question "The MUSICtable system encouraged me to collaborate with others while selection music" was 3.67 (corresponding to an answer of "Neutral-Agree").

The mean response to the survey question "I felt separated from the social atmosphere of the party while participating in music selection using the Winamp system" was 3.125 (corresponding to an answer of "Neutral-Agree"). The mean response to the survey question "I felt separated from the social atmosphere of the party while participating in music selection using the MUSICtable system" was 1.92 (corresponding to an answer of "Strongly Disagree-Disagree").

All users indicated feeling more included in the social circle while using MUSICtable. These results support our hypothesis that the MUSICtable system will preserve social cohesion.

\subsection{Level of Control}

In terms of satisfaction with the level of control over the selection of music, Winamp had the advantage over the MUSICtable. This factor clearly influenced overall system preference: Eight participants indicated a preference for Winamp, three preferred the MUSICtable, and two participants were undecided.

\subsection{Understanding MUSICtable}

The user study indicated problems with the mental model of the system. Many users had trouble understanding how their input affected the selection of music. This stemmed from a lack of understanding of the relationship between the visual map and the music played. Users indicated that the visualization did not provide enough information about which areas of the map corresponded to which type of music.

\subsection{General Feedback}

Other general complaints about the prototype were that users felt that the system progressed too slowly. Participants wanted their input to affect the music being played by the system with more immediacy. Many users expressed a desire to have a "skip" button so that they could progress to the next type of song more quickly - one user commented that he wanted the "instant gratification" of skipping songs. Users also indicated that they lost interest in using the system because of the slow drift of the selection point.

There were aspects of MUSICtable that were well received. Users generally enjoyed the interface and felt that it encouraged participation and led to conversation between people. Some users commented that they felt that the interface was game-like and compelled them to participate and figure out how it worked. People were generally happy with the music played by the MUSICtable and liked that the mood of the music progressed in a continuous manner.

Via the post-experiment survey, users provided ideas about what they would like to see developed in future design iterations. Suggestions included a larger, possibly wall-based display, some sort of preview mode for working out the landscape of music in which only a few seconds of each song is played. Participants indicated a strong desire for clearer labeling of the map as well as the option to skip to the next song. Users also expressed the desire to see the artist name and title of the currently playing song.

\subsection{Discussion}

Our informal user study revealed some interesting observations and provided us with a lot of valuable feedback. Our system made a significant improvement in social interaction, but failed to provide users with the level of control they desired.

The music map visualization that we used in our prototype fell short of expectations in terms of intuitiveness. The landmark pattern chosen to differentiate types of music seemed to detract from the idea of a geographic map. One user asked, "What do the cogs mean?"

We had decided against implementing a "skip" button to allow users to jump to the next song, because it gives any one user too much control and it would disrupt the continuous flow of music. The importance of the skip button is undoubted linked to the traditional Winamp interface where it is a highly used function in the random play mode. We discussed the idea of using a voting system that requires a consensus among users before allowing a song skip. However, the loss of smooth, continuous transition between musical moods would need to be addressed. 
The selection point was designed to drift slowly across the map and the major feedback loop was designed to be the slow progression from song to song. This was a point of frustration for numerous users at our user party. Again, this is an issue of lack of control but we believe that it can be overcome under the right social circumstances. In a party that takes place over the course of an entire night, we believe that users would begin to accept that input is reflected in the progression of music and does not directly change the music.

We did invest considerable thought into some type of labeling of various areas of the map. The table-based aspect of the system complicated this problem, as the display needs to be understandable from any orientation. This was the motivation for a graphical indication of style via edge textures for each category or "island" on the map. Clearly our solution was not intuitive enough, as the majority of users did not understand it. However, our system is designed to be used with any type of music map and with a more intuitive version we believe that users would embrace the idea of exploration. We will have to investigate more effective methods of indicating how the music relates to the map.

\section{FUTURE WORK}

Our informal user study provided a great deal of strong user feedback, which we will incorporate into future revisions of our specific prototype. One area we plan to work on is the development of more music maps to be used with the system. Different collections of music and corresponding visualizations will be effective in different social situations, dependent on the types of users at the party. Improvements to the visualization are needed to better communicate the meaning of different musical regions, allowing for more fluid interaction by users who are unfamiliar with the music map. We also plan to provide a "veto song" voting system that uses the input of multiple users to determine when a song should be skipped. By fading out the skipped song we hope to maintain the feeling of continuity between transitions even with a song skip. As well, we need to tune parameters of the interface, such as the speed of drift of the selection area, to find the most user acceptable parameters. An additional extension to our prototype would provide a layered zoomable map to allow for greater selection control by users.

In addition to improvements to our first prototype we believe that the core concept of our project has very promising alternative designs in various domains.

Portable digital music is becoming a very commonplace commodity with the popularization of personal hardware mp3 players such as the Apple iPod. Our system could be extended to utilize algorithms that automatically classify and organize music collections dynamically. This would allow a guest at a party to download her own collection into the MUSICtable, observing her own specific musical tastes reflected in the map.

The concept of a MUSICtable interface could have very successful application in specific musical domains. Dance halls would be suitable candidates because of the regimented music classification they require. For example, in a ballroom dance hall the types of music are strictly divided based on the specific type of dance (i.e. waltz, rumba, fox trot), and can be further subdivided based on tempo. In this specific social setting, users could plan the progression of dances by specifying a path through the music map interface.
In our prototype design we focused on supporting social collaboration in music selection at a party. The chosen domain assumes that the users are unfamiliar with the music collection and music map displayed on the MUSICtable. The core concept of MUSICtable applied to the domain of single user interaction opens up some interesting possibilities where the user is completely familiar with the music collection and map. Early in the design stage we noted this area as a promising application for the MUSICtable concept. In a single user design, the user could be given finer control over the progression of music, but still maintain the benefit of selecting music above the granularity of the specific song. We envision that the single-user interface could involve tangible interaction by manipulating widgets directly on top of the music table. This type of interaction is not well suited for multi-user collaboration; however, for the single user it has the potential to be a very rich experience. Since the user is completely familiar with all areas of the music map visualization, we also envision an interface in which the user specifies paths through the map to generate a specific time-varying progression of music. In this single user domain, the MUSICtable interface would move from the metaphor of exploration to that of orienteering.

Another future direction of the MUSICtable concept would be to explore the creation of music. Although the MUSICtable may not be effective as a music controller in the traditional sense, we envision that it could be utilized to create music through sampling of pre-recorded music. This type of interface would require a specific organization of music for the performer to be effective in navigating the space and creating a composition out of short music samples.

\section{CONCLUSION}

We have presented a design in which a spatial visualization of a digital music collection is utilized to support exploration and multi-user interaction with music. We implemented a tabletop display prototype to realize this novel interface concept. Informal user testing showed that the design was successful in encouraging collaboration and reducing isolation in music selection tasks within a social environment. Further work is needed to develop a greater level of control and intuitiveness in the interface. However, our design shows promise in terms of extending the utility of map-based visualization of digital music.

\section{REFERENCES}

[1] Gaver, W.W., Bowers, J., Boucher, A., Gellerson, H., Pennington, S., Schmidt, A., Steed, A., Villars, N., Walker, B. The drift table: designing for ludic engagement. In Proc CHI '04 extended abstracts on Human factors in computing systems, ACM Press (2004), 885 - 900.Ding, W., and Marchionini, G. A Study on Video Browsing Strategies. Technical Report UMIACS-TR-97-40, University of Maryland, College Park, MD, 1997.

[2] van Gulik, R., Vignoli, F., and van de Wetering, H. Mapping music in the palm of your hand, explore and discover your collection. ISMIR 2004 5th International Conference on Music Information Retrieval. Available at: http://www.iua.upf.es/mtg/ismir2004/review/CRFILES/paper 153-fac43d9ee7ddd1b5d0f50b5d35008a77.pdf

[3] Mazalek, A., and Jehan, T. Interacting with Music in a Social Setting. Proc. CHI '00 extended abstracts on Human factors in computing systems, ACM Press (2000), 255 - 256. 
[4] O’Hara, K., Lipson, M., Jansen, M., Unger, A., Jeffries, H., and Macer, P. Jukola: Democratic Music Choice in a Public Space. Proc. DIS2004, ACM Press (2004), 145 - 154.

[5] Pampalk, E., Rauber, A., and Merkl, D. Content-based organization and visualization of music archives. Proc. ACM Multimedia 2002, ACM Press (2002), 570 - 579.

[6] Rogers, Y., and Lindley, S. (2003). Collaborating around large interactive displays: which way is best to meet? Available at:

http://www.informatics.sussex.ac.uk/interact/papers/pdfs/per
vasive_environments_and_ubiComp/Shared_interaction_spa ces/Rogers.displays2003.p̄ff

[7] Torrens, M., Hertzog, P., and Arcos, J. Visualizing and exploring personal music libraries. ISMIR 2004 5th International Conference on Music Information Retrieval. Available at:

http://www.iua.upf.es/mtg/ismir2004/review/CRFILES/paper 214-86063c9f6c9c3debfdccb432405ffa47.pdf

[8] Weiser, M. (1991), The computer for the twenty-first century, Scientific American 265(3), 94 - 104.

[9] Nullsoft Winamp. http://www.winamp.com 